\title{
-Short Communicaotion-
}

\section{Antigenic analysis of influenza viruses isolated in Thailand in the rainy season, 1989}

\author{
Yasue Takeuchi ${ }^{1)}$, Kazuyuki Nakamura ${ }^{2)}$, Suwicha Kupradinunt ${ }^{3)}$ \\ and Yukio Yamazi4) \\ 1) Chiba Prefectural Serum Institute, Chiba \\ 2) Nagano Research Institute for Health and Pollution, Nagano \\ 3) National Institute of Health, Nongtabri, Thailand \\ 4) Nippon Medical School, Tokyo
}

In the rainy season of 1989,72 strains of influenza viruses were isolated in Thailand by using MDCK cell cultures, which included 36 samples belonging to H1N1, 24 samples belonging to H3N2 subtype of type $A$ and 12 samples of type $B^{1}$. The identified viruses were inoculated into developing hen's eggs of 10 days' age, and $22(32.6 \%)$ of them were adapted to the egg after one passage, which included $8 / 36(22.2 \%)$ of $\mathrm{H} 1 \mathrm{~N} 1,7 / 18(38.2 \%)$ of $\mathrm{H} 3 \mathrm{~N} 2$ and $7 / 8(87.5 \%)$ of type B virus. The adapted viruses were analyzed antigenically with the haemagglutination inhibition (HI) test using mouse antisera raised to 16 reference viruses shown in Table 1 and 2.

As shown in Table 1, among eight H1N1 strains isolated in Chiang Mai in 1989, four (A/Chiang Mai/28, 71, 73 and 105/89) (50\%) were antigenically similar to A/Yamagata/32/89 as well as to $\mathrm{A} / \mathrm{Chiang} \mathrm{Mai} / 39$ or $101 / 87$ (i.e., any differences of $\mathrm{HI}$ titers from those of A/Yamagata/32/89 and A/Chiang Mai/39 and 101/89 were within two folds), one (A/Chiang Mai/120/89) (13\%) was similar to A/Yamagata/120/86 (i.e., any difference of HI titer was within 2 folds), and the other three (A/Chiang Mai/27, 62 and 108/89) (38\%) were antigenically not so different from A/Chiang Mai/39/87 or A/Yamagata/32/89 (i.e., only four folds differences of the HI titers to anti-A/Chiang Mai/39 and/or 101/89 sera were observed). Thus, seven out of eight strains (87.5\%) of H1N1 influenza virus isolated in Chiang Mai in August, 1989 were antigenically closely related to what prevailed in Chiang Mai in 1987 and in Japan in 1989, suggesting that the antigenic variation of H1N1 subtype in Thailand and in Japan was minor during 1987 to 1989. Incidentally H1N1 subtype of the virus has not been isolated in Japan from May, 1989 until February, 1991'2), but 11 strains of the subtype were isolated in Chiang Mai in August 19903).

As shown in Table 1, among 10 H3N2 strains, seven (A/Bangkok/235, 238 and 246/89 and A/Chiang Mai/9, 52, 80 and 86/89) (70\%) were antigenically similar to A/Akita/4/88 which was equivalent to $\mathrm{A} / \mathrm{Hokkaido} / 20 / 89$ (i.e., any difference of $\mathrm{HI}$ titer was within two folds), two (A/Chiang Mai/48 and 111/89) (20\%) were similar to A/Sichuan/2/87 (i.e., any difference of HI titer was within two folds), and the other one (A/Chiang Mai/51/89) (10\%) was antigenically not so different from A/Akita/4/88 (i.e., difference of HI titer was within two folds, except that to anti-A/Fukuoka/c-29/85 serum which was four folds). According to an antigenic analysis of H3N2 subtype isolated in Japan (Influenza Survey of 1989 1990 Season by NIH, Tokyo) ${ }^{21}, 67 \%$ out of 502 
Table 1 Haemagglutination inhibition reaction of type A influenza viruses isolated in Thailand in July and August, 1989

\begin{tabular}{|c|c|c|c|c|c|c|}
\hline \multirow{15}{*}{$\begin{array}{l}\frac{8}{2} \\
\text { 泀 } \\
\overrightarrow{2} \\
\vec{z} \\
\vec{z}\end{array}$} & \multirow[b]{2}{*}{ Virus } & \multicolumn{5}{|c|}{ Mouse antiserum raised to: } \\
\hline & & $\underset{10 / 83}{\mathrm{~A} / \text { Bangkok/ }}$ & $\underset{120 / 86}{\text { A/Yamagata/ }}$ & $\underset{39 / 87}{\text { A/Chiang Mai/ }}$ & $\begin{array}{c}\text { A/Chiang } \\
101 / 87\end{array}$ & A/Yamagata/ \\
\hline & A/Bangkok/10/83 & $\underline{128}$ & 64 & $<32$ & $<32$ & $<32$ \\
\hline & A/Yamagata/120/86 & 64 & $\underline{2,048}$ & 512 & 512 & 512 \\
\hline & A/Chiang Mai/39/87 & 32 & 1,024 & 256 & 64 & 512 \\
\hline & A/Chiang Mai/101/87 & $<32$ & 1.024 & 128 & $\underline{512}$ & 256 \\
\hline & A/Yamagata/32/89 & $<32$ & 1,024 & 128 & $\overline{256}$ & $\underline{512}$ \\
\hline & A/Chiang Mai/27/89 & $<32$ & 512 & 64 & 64 & 512 \\
\hline & A/Chiang Mai/28/89 & 32 & 2,048 & 256 & 512 & 512 \\
\hline & A/Chiang $\mathrm{Mai} / 62 / 89$ & $<32$ & 512 & 64 & 64 & 256 \\
\hline & A/Chiang $\mathrm{Mai} / 71 / 89$ & $<32$ & 1,024 & 256 & 128 & 256 \\
\hline & A/Chiang Mai/73/89 & $<32$ & 1,024 & 128 & 256 & 256 \\
\hline & A/Chiang Mai/105/89 & $<32$ & 1,024 & 128 & 256 & 256 \\
\hline & A/Chiang Mai/108/89 & $<32$ & 512 & 128 & 64 & 128 \\
\hline & A/Chiang Mai/120/89 & 64 & 2,048 & 256 & 256 & 256 \\
\hline \multirow{16}{*}{ 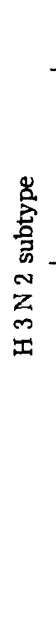 } & & $\begin{array}{c}\text { A/Niigata/ } \\
102 / 81\end{array}$ & A/Philippines/ & $\begin{array}{c}\text { A/Fukuoka/ } \\
\text { C-29/85 }\end{array}$ & $\begin{array}{l}\text { A/Sichuan/ } \\
2 / 87\end{array}$ & $\begin{array}{c}\text { A/Akita/ } \\
4 / 88\end{array}$ \\
\hline & A/Niigata/102/81 & $\underline{256}$ & 128 & 64 & $<32$ & $<32$ \\
\hline & A/Philippines/2/82 & $<32$ & $\underline{256}$ & $<32$ & $<32$ & $<32$ \\
\hline & A/Fukuoka/C-29/85 & $<32$ & $<32$ & $\underline{2,048}$ & 128 & 64 \\
\hline & A/Sichuan/2/87 & $<32$ & $<32$ & 32 & 1,024 & 64 \\
\hline & A/Akita/4/88 & $<32$ & 64 & 256 & $\underline{256}$ & $\underline{1,024}$ \\
\hline & A/Bangkok/235/89 & $<32$ & 32 & 128 & 128 & 512 \\
\hline & A/Bangkok/238/89 & $<32$ & 32 & 128 & 512 & 512 \\
\hline & A/Bangkok/246/89 & $<32$ & 32 & 128 & 128 & 512 \\
\hline & A/Chiang Mai/9/89 & $<32$ & 32 & 128 & 128 & 512 \\
\hline & A/Chiang Mai/48/89 & $<32$ & 32 & 64 & 1,024 & 64 \\
\hline & A/Chiang Mai/51/89 & $<32$ & 32 & 64 & 128 & 512 \\
\hline & A/Chiang Mai/52/89 & $<32$ & 64 & 256 & 256 & 1,024 \\
\hline & A/Chiang Mai/80/89 & 32 & 64 & 256 & 512 & 1,024 \\
\hline & A/Chiang Mai/86/89 & 32 & 64 & 256 & 512 & 1,024 \\
\hline & A/Chiang Mai/111/89 & 32 & $<32$ & 32 & 512 & 64 \\
\hline
\end{tabular}

strains were antigenically similar to $\mathrm{A} / \mathrm{Hokkaido} / 20 / 89$ and $28 \%$ were similar to $\mathrm{A} / \mathrm{Sichuan} / 2-87$ (i.e., any differences of $\mathrm{HI}$ titers were within two folds). In other words, no definite antigenic change of H3N2 subtype of the virus was observed in Thailand and in Japan in 1989.

As shown in Table 2, all of the six type B isolates were antigenically similar (i.e., any difference of $\mathrm{HI}$ titer was within two folds) to B/Yamagata/16/88 which was antigenically very different from type B viruses prevailed before 1988. Influenza epidemics in Japan in the 1989/90 season were caused by H3N2 subtype and type B viruses, and $89 \%$ of type B isolates in Japan in the $1989 / 90$ season were antigenically similar to B/Yamagata/16/882). Therefore, definite antigenic difference of type B virus was not observed between Japan and Thailand. 
Table 2 Haemagglutination inhibition reaction of type B influenza viruses isolated in Thailand in July and August, 1989

\begin{tabular}{|c|c|c|c|c|c|c|}
\hline \multirow{2}{*}{ Virus } & \multicolumn{6}{|c|}{ Mouse antiserum raised to: } \\
\hline & $\begin{array}{c}\text { B/USSR/ } \\
100 / 83\end{array}$ & $\begin{array}{c}\text { B/Chiang Mai/ } \\
5 / 87\end{array}$ & B/Chiang Mai/ & $\begin{array}{c}\text { B/Nagasaki/ } \\
1 / 87\end{array}$ & $\begin{array}{l}\text { B/Aichi/ } \\
5 / 88\end{array}$ & B/Yamagata/ \\
\hline B/USSR/100/83 & $\underline{512}$ & 16 & $<16$ & 16 & $<16$ & $<16$ \\
\hline B/Chiang Mai/5/87 & $\overline{16}$ & $\underline{512}$ & 32 & 64 & 32 & $<16$ \\
\hline B/Chiang Mai/45/87 & 16 & 128 & $\underline{128}$ & 128 & 16 & $<16$ \\
\hline B/Nagasaki/1/87 & 128 & 512 & $\overline{128}$ & $\underline{512}$ & 128 & $<16$ \\
\hline B/Aichi/5/88 & 64 & 512 & 64 & $\overline{128}$ & $\underline{128}$ & $<16$ \\
\hline B/Yamagata/16/88 & 32 & 16 & 16 & 32 & 16 & $\underline{1,024}$ \\
\hline B/Bangkok/131/89 & 32 & 16 & 16 & 64 & 16 & 1,024 \\
\hline B/Bangkok/258/89 & 16 & 16 & 16 & 16 & $<16$ & 1,024 \\
\hline B/Chiang Mai/26/89 & 16 & $<16$ & 16 & 32 & $<16$ & 512 \\
\hline B/Chiang Mai/36/89 & $<32$ & 16 & 16 & 32 & 16 & 512 \\
\hline B/Chiang Mai/76/89 & 16 & $<16$ & 16 & 16 & 16 & 512 \\
\hline B/Chiang Mai/96/89 & 16 & 16 & 16 & 16 & 16 & 512 \\
\hline $\mathrm{B} /$ Chiang $\mathrm{Mai} / 117 / 89$ & 16 & $<16$ & 16 & 16 & $<16$ & 512 \\
\hline
\end{tabular}

\section{References}

1) Takeuchi, Y., Nakayama, K., Kupradinunt, S., Peerakome, S., Supawadee, J., Suprasert, S., Hara, M., Damrongsak, D., Vithayasai, V. and Yamazi, Y.: Nippon Ika Daigaku Zashi, 57, 556, 1990.

2) Monthly Report on Findings of Infectious Agents in Japan., 11, 237, 1990 (in Japanese).

3) Takeuchi, Y., Nakayama, K., Ohde, H., Yamazi, Y., Pongprot, B., Suprasert, S., Supawadee, J., Vithayasai, V. and Damrongsak, D.: Report from the 20th Medical Research Team of the Society for Medical Research in South-East Asia, Nippon Medical School, 1991 (in Japanese). 